\title{
Robust Spectrum Sensing based on Statistical Tests
}

\author{
Kamran Arshad ${ }^{\dagger}$ and Klaus Moessner ${ }^{\ddagger}$, \\ ${ }^{\dagger}$ School of Engineering, University of Greenwich, United Kingdom \\ ${ }^{\ddagger}$ Centre for Communication Systems Research, University of Surrey, United Kingdom \\ Email: k.arshad@greenwich.ac.uk
}

\begin{abstract}
Spectrum sensing, in particular, detecting the presence of licensed or incumbent users in licensed spectrum, is one of the pivotal tasks in cognitive radio network. In this paper, we tackle the spectrum sensing problem by using statistical test theory and derive novel spectrum sensing approaches. We apply the classical Kolmogorov-Smirnov (KS) test under the assumption that the noise probability distribution is known. However, as in practice, the exact noise distribution is unknown, a sensing method for Gaussian noise with unknown noise power is proposed in this article and refer as $t$-sensing. The proposed sensing scheme is asymptotically robust and can be applied to non-Gaussian noise distributions. A closed form equation determining the miss detection probability for the $t$-sensing is derived. We compare the performance of our sensing algorithms with the Energy Detector (ED) and Anderson-Darling (AD) sensing proposed in literature. Simulation results show that the proposed sensing methods outperform both $\mathrm{ED}$ and $\mathrm{AD}$ based sensing, especially for the case when the received signal to noise ratio is low.
\end{abstract}

\section{Index Terms}

Spectrum sensing, Cognitive radio, Non-parametric detection

\section{INTRODUCTION}

A cognitive or opportunistic user (OU) must be aware and perceptive of its operating environment, which makes spectrum sensing one of the most important operations for the realisation of cognitive radio networks [1]. The objectives of spectrum sensing are twofold: (1) to detect and protect incumbent users referred here as IUs and (2) to exploit unused portions of spectrum ("whitespaces") not only to enhance the Quality of Service (QoS) of OUs but also to provide opportunistic usage of the spectrum. In the 2010 
Federal Communications Commission (FCC) ruling [2], a geo-location database based query mechanism was proposed for the protection of IUs and the FCC significantly weakened the sensing requirement for TV Band Devices (TVBDs), initially required, back in 2008 [3]. However, spectrum regulators, including the Ofcom in UK, FCC in USA and other European regulatory agencies represented by the European Conference of Postal and Telecommunications Administrations (CEPT), continue to encourage researchers to investigate on spectrum sensing techniques [4].

Detecting either incumbent or other opportunistic users in a certain frequency band is a challenging task for many reasons [2]. A number of spectrum sensing algorithms have been proposed in literature and each algorithm has its own operational requirements, advantages and disadvantages; a comprehensive survey has been published by Yucek et al [5]. Spectrum sensing algorithm based on Anderson Darling (AD) test has been proposed in [6]. The authors consider the spectrum sensing problem as a goodness-offit (GoF) testing problem and used the AD test to check whether the received signal samples were drawn from the known noise distribution. From a mathematical point of view, the AD test is asymptotically distribution-free which means that for large sample sizes, the decision threshold is independent of the noise and can be calculated in advance for any arbitrary noise distribution. Our simulations show that AD sensing performs superior to energy detection (ED) sensing for the system model defined in [6] i.e. detection of non-zero mean in Gaussian noise. However, in most other scenarios the variance of received signal is always different than the variance of the noise distribution.

In this paper, we assume that signals received by an OU have non-zero means and a variance that is different from the variance of noise. In certain communication applications, non-zero mean signals appear in a natural way e.g. double-sideband amplitude modulation carrier format, frequency shift keying with large frequency deviation etc [7]. We investigate the spectrum sensing problem by exploiting the theory of statistical testing. In particular, we propose a sensing scheme derived from the modified Student's $t$-test ( $t$-sensing). A two sample version of KS test has already been applied to the problem of spectrum sensing in [8]. In this article, we also generalize KS sensing without holding the assumption of having two-samples and compare the detection performance with AD sensing proposed in [6]. Our proposed schemes do not require a-priori knowledge of the incumbent (or opportunistic) user signal and hence we classify them as non-parametric schemes. We compare the sensing performance of our methods with the well-known ED sensing and AD sensing proposed in [6]. For KS sensing we used the assumption that the noise probability distribution is known to the user, as in [6]. Our simulation results show that under similar conditions and assumptions as in [6], KS sensing gives better sensing performance than AD sensing. 
Our alternative sensing scheme, $t$-sensing, does not require a-priori knowledge of the IU signal nor the noise density function. However, it needs a short sequence of noise samples which can be measured from a reporting channel (where it is sure that a transmitted signal is not present). It should be noted that this assumption is essentially the same as used in the well known ED (and other sensing algorithms like AD sensing [6]). Our scheme, $t$-sensing, compares the received signal samples with the noise samples and makes a decision. We derive the test statistics of $t$-sensing with the assumption that the noise process is Gaussian. Exploiting simulation results, we show that $t$-sensing is asymptotically robust and can be applied to any arbitrary noise distribution. Numerical results show that $t$-sensing performs much better than $\mathrm{AD}$ and $\mathrm{ED}$ sensing and leads to low computational complexity particularly at low SNR values.

This article is organised as follows. In section II we describe the system model and formulate the spectrum sensing problem as a hypothesis-testing problem. Spectrum sensing using statistical tests is explained in section III. In section IV, the performance of the proposed sensing schemes are evaluated numerically and compared with existing sensing algorithms. Conclusions and recommendations are provided in section $\mathrm{V}$. We use following notations in this article; all column vectors are represented by boldface letters, $F(X)$ and $f(X)$ denote the distribution and density function of random variable $X$ respectively and $\Phi($.$) represents the distribution function of the standard Gaussian random process.$

\section{SySTEM MODEL}

Suppose that the bandwidth of interest for an OU is $W$, which is scanned in observation time $T$. Let $N$ be an integer close to $T \times W$. We then formulate the spectrum sensing problem as a binary hypothesis testing problem as follows:

$$
z_{i}= \begin{cases}n_{i} & ; \mathcal{H}_{0} \\ C h_{i} s_{i}+n_{i}, & ; \mathcal{H}_{1}\end{cases}
$$

where $i \in \mathcal{S}=\{1,2, \ldots, N\}, C$ represents the fixed Received Signal Strength (RSS) at the OU, $n_{i}$ is the complex Gaussian noise sample of zero mean and variance $\sigma_{n}^{2}, h_{i}$ is a complex Gaussian random variable that represents flat fading of the channel with mean $\mu_{h}$ and variance $\sigma_{h}^{2}$, and $s_{i}$ is the transmit signal through a Rician channel. We use the term transmit signal for either the signal transmitted by incumbent or other opportunistic users. In addition to the path loss, RSS $(C)$ also encapsulates any shadowing effects that the transmit signal may experience at the receiver. Further, $\mathcal{H}_{0}$ represents the hypothesis that only noise is present, and $\mathcal{H}_{1}$ represents the alternate hypothesis that a transmit signal and noise are present. 
Define

$$
\mathrm{SNR}=\frac{C^{2}\left(\left|\mu_{h}^{2}\right|+\sigma_{h}^{2}\right)}{\sigma_{n}^{2}},
$$

and $K=\frac{\left|\mu_{h}^{2}\right|}{\sigma_{h}^{2}}$. Let $\mathbf{z}=\left[z_{1}, z_{2}, \cdots, z_{N}\right]^{\mathrm{T}}$ and $\mathbf{y}=\left[y_{1}, y_{2}, \cdots, y_{N}\right]^{\mathrm{T}}=\operatorname{sort}(\mathbf{z})$ where $\operatorname{sort}($.$) represents$ the sorting function such that $y_{1} \leqslant y_{2} \leqslant y_{3} \leqslant \cdots \leqslant y_{N}$. Let $\sigma_{y}^{2}$ be the variance of $y_{i}$ and it is given as $\sigma_{y}^{2}=C^{2} \sigma_{h}^{2}+\sigma_{n}^{2}=\sigma_{n}^{2}(1+\mathrm{SNR} /(1+K))$. We assume that noise samples $n_{i}$ and $n_{j}$ for all $i, j \in \mathcal{S}$ are independent of each other and the Cumulative Distribution Function (CDF) of the noise process is continuous. The exact form of distribution of $\mathbf{y}$ under $\mathcal{H}_{1}$ depends on the channel conditions as well as on the noise distribution, for the case of AWGN and Rician fading channel the following applies:

$$
\begin{aligned}
& f\left(\mathbf{y} \mid \mathcal{H}_{0}\right)=\left(2 \pi \sigma_{n}^{2}\right)^{-N / 2} \exp \left\{-\sum_{i \in \mathcal{S}} \frac{y_{i}^{2}}{2 \sigma_{n}^{2}}\right\} \\
& f\left(\mathbf{y} \mid \mathcal{H}_{1}\right)=\left(\pi \sigma_{y}^{2}\right)^{-N} \exp \left\{-\frac{1}{\sigma_{y}^{2}} \sum_{i \in \mathcal{S}}\left|y_{i}-C \mu_{h}\right|^{2}\right\},
\end{aligned}
$$

Let $f(\mathbf{y})$ and $F(\mathbf{y})$ are the empirical density and distribution function of the received signal $\mathbf{y}$ and

$$
F(\mathbf{y})=\frac{1}{N} \sum_{i \in \mathcal{S}} I_{y_{i} \leqslant y},
$$

where $I_{a \leqslant b}$ is the indicator function and is defined as

$$
I_{a \leqslant b}= \begin{cases}1 & \text { if } a \leqslant b \\ 0 & \text { otherwise. }\end{cases}
$$

For any spectrum sensing algorithm, first a test statistic is calculated; defined as $\theta(\mathbf{y})$. This test statistic is then compared with a decision threshold $\lambda$ to decide if the transmit signal is present; $\theta(\mathbf{y})>\lambda$ indicates that a transmit signal is present and vice versa. The Receiver Operating Characteristics (ROC) curve is an important metric to evaluate the performance of a spectrum sensing algorithm [1]. The ROC curves that we consider in this paper plots probability of miss-detection $P_{m}$ (or detection $P_{d}$ ) versus probability of false alarm $P_{f}$ and defined as

$$
\begin{gathered}
P_{f}=\operatorname{Prob}\left\{\theta(\mathbf{y})>\lambda \mid \mathcal{H}_{0}\right\}, \\
P_{m}=\operatorname{Prob}\left\{\theta(\mathbf{y})<\lambda \mid \mathcal{H}_{1}\right\},
\end{gathered}
$$

Energy detection is a common method to detect an unknown signal in AWGN channel [1]. For ED sensing decision rule is given by

$$
\theta_{\mathrm{ED}} \triangleq \frac{1}{N} \sum_{i \in \mathcal{S}}|y(i)|^{2} \underset{\mathcal{H}_{0}}{\stackrel{\mathcal{H}_{1}}{\gtrless}} \lambda_{\mathrm{ED}},
$$


where $\theta_{\mathrm{ED}}$ and $\lambda_{\mathrm{ED}}$ represents test statistics and decision threshold for ED sensing. Based on the decision rule defined in equation (9), $P_{f}$ and $P_{d}$ for ED sensing can be derived as [9]

$$
\begin{aligned}
P_{f} & =\frac{\Gamma\left(N, \lambda_{\mathrm{ED}} / 2\right)}{\Gamma(N)} \triangleq \mathcal{G}_{N}\left(\lambda_{\mathrm{ED}}\right), \\
P_{d} & =Q_{m}\left(\sqrt{2 N \cdot \mathrm{SNR}}, \sqrt{\mathcal{G}_{N}^{-1}\left(P_{f}\right)}\right),
\end{aligned}
$$

where $\Gamma(a)$ is the gamma function, $\Gamma(a, x)$ is the incomplete gamma function and $Q_{m}(a, b)$ is the generalised Marcum $Q$-function.

\section{SPECTRUM SENSING BASED ON STATISTICAL TESTS}

The main purpose of spectrum sensing is to obtain awareness about the spectrum usage and the existence of transmitting users in a given geographical area. In many practical situations, the knowledge of incumbent and/or other OU signals is limited, incomplete or imprecise. Hence, a sensor which is designed for a specific signal type (i.e. waveform) may suffer drastic degradation in performance when sensing other signals. Proposed spectrum sensing methods using statistical tests are more robust in a sense that they are independent of the transmit signal. When there is no signal transmission, the received signal $\mathbf{y}$ is a sequence of samples drawn independently from the noise $f\left(\mathbf{y} \mid \mathcal{H}_{0}\right)$. Similarly, under $\mathcal{H}_{1}$, the received samples are drawn from the density $f\left(\mathbf{y} \mid \mathcal{H}_{1}\right)$, defined in equation (4), such that $f\left(\mathbf{y} \mid \mathcal{H}_{0}\right) \neq f\left(\mathbf{y} \mid \mathcal{H}_{1}\right)$. Hence, the spectrum sensing problem can be transformed into a simple problem of testing the hypothesis $\mathcal{H}_{0}$; if we cannot reject $\mathcal{H}_{0}$ under the given constraints, then $\mathcal{H}_{0}$ is retained and the channel is available for opportunistic use.

In the following, we describe spectrum sensing algorithms based on statistical tests. We first summarize the general steps in making a decision about the existence of a transmit signal in a given frequency band $W$. This is followed by an introduction to $\mathrm{AD}$ sensing. We then propose and describe KS sensing under the assumption that the noise probability density function is known, that is, $f\left(\mathbf{y} \mid \mathcal{H}_{0}\right)$ is an arbitrary (but otherwise known) distribution. Later, we relax the assumption of the known noise density, and explain $t$-sensing which is asymptotically robust for any noise distribution. Simulation results show then that $t$-sensing in non-Gaussian noise gives similar performance as sensing in the presence of Gaussian noise. The general steps for spectrum sensing for any test can be summarised as follows:

1) Sort the received samples in ascending order (not necessary in ED and $t$-sensing).

2) Calculate the threshold $\lambda$ for a given probability of false alarm, say $\widetilde{P}_{f}$.

3) Calculate the test statistic $\theta$ for a given test.

4) Compare $\theta$ with $\lambda$ to make a decision about the existence of transmit signal. 


\section{A. AD Sensing}

The AD test is used to check whether the received samples at an OU are drawn from a known noise distribution $F\left(\mathbf{y} \mid \mathcal{H}_{0}\right)$. The AD test statistic is given by [10]

$$
\theta_{\mathrm{AD}} \triangleq N \times \int_{-\infty}^{+\infty}\left[F(\mathbf{y})-F\left(\mathbf{y} \mid \mathcal{H}_{0}\right)\right]^{2} \psi\left(F\left(\mathbf{y} \mid \mathcal{H}_{0}\right)\right) \mathrm{d} F\left(\mathbf{y} \mid \mathcal{H}_{0}\right),
$$

where $\psi(u)=(u(1-u))^{-1}$ is a weight function defined over $0 \leqslant u<1$. The integral in equation (11) can be performed and a simplified form of $\theta_{\mathrm{AD}}$ is obtained as

$$
\theta_{\mathrm{AD}}=-\frac{\left(N^{2}+\sum_{i \in \mathcal{S}}(2 i-1)\left(\ln Z_{i}+\ln \left[1-Z_{1+N-i}\right]\right)\right)}{N},
$$

where $Z_{i}=F\left(y_{i} \mid \mathcal{H}_{0}\right)$. The asymptotic distribution of $\theta_{\mathrm{AD}}$ under $\mathcal{H}_{0}$ can be derived as [10]

$$
\begin{aligned}
F\left(\theta_{\mathrm{AD}} \mid \mathcal{H}_{0} ; x\right)= & \frac{\sqrt{2 \pi}}{x} \sum_{j=0}^{+\infty} a_{j}(4 j+1) \exp \left(-\frac{(4 j+1)^{2} \pi^{2}}{8 x}\right) \\
& \int_{0}^{+\infty}\left(\frac{x}{8\left(w^{2}+1\right)}-\frac{(4 j+1)^{2} \pi^{2} w^{2}}{8 x}\right) \mathrm{d} w,
\end{aligned}
$$

where $a_{j}=(-1)^{j} \Gamma\left(j+\frac{1}{2}\right) /\left(\Gamma\left(\frac{1}{2}\right) j !\right)$. From equation (7) and (13), the threshold $\lambda_{\mathrm{AD}}$ can be calculated for a given $\widetilde{P}_{f}$ using equation (14)

$$
\widetilde{P}_{f}=1-F\left(\theta_{\mathrm{AD}} \mid \mathcal{H}_{0} ; \lambda_{\mathrm{AD}}\right) .
$$

Hence, for large $N$, equations (13) and (14) give the thresholds for different values of probability of false alarm, a table is given in [11]. Finally, $\theta_{\mathrm{AD}}$ is compared with $\lambda_{\mathrm{AD}}$ to check if $\mathcal{H}_{0}$ can be rejected at a given $\widetilde{P}_{f}$ and a decision is made as follows: if $\theta_{\mathrm{AD}}>\lambda_{\mathrm{AD}}$ the hypothesis $\mathcal{H}_{0}$ is rejected and the channel is considered as occupied; alternatively the $\mathcal{H}_{0}$ is accepted and the channel is assumed to be unoccupied.

\section{B. KS Sensing}

Like the $\mathrm{AD}$ test, the $\mathrm{KS}$ test also evaluates the hypothesis that received signal samples, $\mathbf{y}$, with empirical distribution function $F(\mathbf{y})$ are drawn from a given distribution function of noise; that is, $F\left(\mathbf{y} \mid \mathcal{H}_{0}\right)$. Under the same assumption that the noise distribution is known to the user, we compare the estimated CDF of received signal with the given CDF of noise. Graphically, the KS test statistic is the maximum vertical distance between the two distributions; that is, $F(\mathbf{y})$ and $F\left(\mathbf{y} \mid \mathcal{H}_{0}\right)$.

$$
\theta_{\mathrm{KS}}=\sup _{\mathbf{y}}\left|F\left(\mathbf{y} \mid \mathcal{H}_{0}\right)-F(\mathbf{y})\right| .
$$


The distribution-free property of the KS test means that $\theta_{\mathrm{KS}}$ has a distribution function that is independent of the distribution of noise under $\mathcal{H}_{0}$. In [12], there are formulas to calculate test statistics for small values of $N$ and also for the asymptotic distribution as $N \rightarrow \infty$ under $\mathcal{H}_{0}:$

$$
\operatorname{Prob}\left(\sqrt{N} \theta_{\mathrm{KS}} \leqslant \lambda_{\mathrm{KS}}\right)=\sum_{j=-\infty}^{+\infty}(-1)^{j} \exp \left(-2 j^{2} \lambda_{\mathrm{KS}}{ }^{2}\right) .
$$

According to the Glivenko-Cantelli theorem, under the null hypothesis $\mathcal{H}_{0}$, the test statistic tends towards zero as

$$
\operatorname{Prob}\left(\lim _{N \rightarrow \infty} \theta_{\mathrm{KS}}=0\right)=1,
$$

which proves that the test will reject a false hypothesis with probability 1 as more and more samples are included to calculate the test statistic $\theta_{\mathrm{KS}}$.

From equations (7) and (16), the threshold for KS sensing for a given $\widetilde{P}_{f}$ can be calculated as

$$
\widetilde{P}_{f}=1-F\left(\theta_{\mathrm{KS}} \mid \mathcal{H}_{0} ; \lambda_{\mathrm{KS}}\right)
$$

Massey [12] provides tables and recursive expressions to calculate the threshold $\lambda_{\mathrm{KS}}$ for a given $\widetilde{P}_{f}$ using equation (18).

\section{C. $t$-Sensing}

Thermal noise in communication channels is widely modelled as an AWGN process with zero mean and unknown variance. Under the hypothesis $\mathcal{H}_{1}$, the probability density function of the received signal is dependent on the channel conditions and the distribution of noise, as defined in equation (4). According to the signal detection theory, the optimal test for detecting non-zero mean signal in a zero mean noise process is the Students t-test [13]. Students t-test can not be applied directly to the spectrum sensing problem as the variance of the received signal under $\mathcal{H}_{1}$ is significantly different from the variance of noise process. Hence, we applied a modified form of $t$-test to our problem. We assume noise samples can be collected from a reporting channel and are treated as reference samples to make a decision. As indicated in section I, reference noise samples can be collected during the system dead time or on a control channel and stored in the memory for the detection process.

Assume that the size of noise samples is the same as for data samples i.e. $N$ and denoted by $\mathbf{x}=$ $\left[x_{1}, x_{2}, \cdots, x_{N}\right]^{\mathrm{T}}$ having cumulative function $F(\mathbf{x})$ and density function $f(\mathbf{x})$. Since both $f(\mathbf{x})$ and $f(\mathbf{y})$ 
are Gaussian and independent, the joint density function, given $\mathcal{H}_{0}$ is true, is written as

$$
\begin{aligned}
\mathfrak{g}\left(\mathbf{x}, \mathbf{y} \mid \mathcal{H}_{0}\right) & =f(\mathbf{x}) \times f\left(\mathbf{y} \mid \mathcal{H}_{0}\right) \\
& =\left(2 \pi \sigma_{n}^{2}\right)^{-N} \exp \left(-\frac{\sum_{i \in \mathcal{S}} x_{i}^{2}+\sum_{i \in \mathcal{S}} y_{i}^{2}}{2 \sigma_{n}^{2}}\right) .
\end{aligned}
$$

Similarly, if $\mathcal{H}_{1}$ is true

$$
\begin{aligned}
\mathfrak{g}\left(\mathbf{x}, \mathbf{y} \mid \mathcal{H}_{1}\right) & =f(\mathbf{x}) \times f\left(\mathbf{y} \mid \mathcal{H}_{1}\right) \\
& =\left[2 \pi\left(\sigma_{n}^{2}+\sigma_{y}^{2}\right)\right]^{-N} \exp \left(-\frac{\sum_{i \in \mathcal{S}} x_{i}^{2}}{2 \sigma_{n}^{2}}-\frac{\sum_{i \in \mathcal{S}}\left(y_{i}-\tilde{\mu}\right)^{2}}{2 \sigma_{y}^{2}}\right) .
\end{aligned}
$$

At the OU the parameters $\tilde{\mu}=C \mu_{h}, \sigma_{n}^{2}$ and $\sigma_{h}^{2}$ are unknown, hence, in order to derive test statistic using the likelihood ratio, these parameters need to be estimated. This estimation can be done by solving:

$$
\begin{gathered}
\frac{\partial}{\partial \sigma_{n}^{2}} \ln \mathfrak{g}\left(\mathbf{x}, \mathbf{y} \mid \mathcal{H}_{0}\right)=0 \\
\frac{\partial}{\partial \tilde{\mu}} \ln \mathfrak{g}\left(\mathbf{x}, \mathbf{y} \mid \mathcal{H}_{1}\right)=0 \\
\frac{\partial}{\partial \sigma_{h}^{2}} \ln \mathfrak{g}\left(\mathbf{x}, \mathbf{y} \mid \mathcal{H}_{1}\right)=0
\end{gathered}
$$

Solution of equation (21) yields the maximum value of the density function as

$$
\max _{\sigma_{n}^{2}} \mathfrak{g}\left(\mathbf{x}, \mathbf{y} \mid \mathcal{H}_{0}\right) \quad \text { and } \max _{\tilde{\mu}, \sigma_{h}^{2}} \mathfrak{g}\left(\mathbf{x}, \mathbf{y} \mid \mathcal{H}_{1}\right) .
$$

The log likelihood ratio is then calculated as

$$
\mathcal{L}(\mathbf{x}, \mathbf{y})=\frac{\mathfrak{g}\left(\mathbf{x}, \mathbf{y} \mid \mathcal{H}_{1}\right)}{\mathfrak{g}\left(\mathbf{x}, \mathbf{y} \mid \mathcal{H}_{0}\right)}
$$

Substituting values from (22) in (23), the test statistic for $t$-sensing can be written as follows

$$
\theta_{t}=\left\{1+\frac{\left(\sum_{i \in \mathcal{S}} y_{i}-\sum_{i \in \mathcal{S}} x_{i}\right)^{2}}{2 N\left[\sum_{i \in \mathcal{S}}\left(x_{i}-\sum_{i \in \mathcal{S}} x_{i} / N\right)^{2}+\sum_{i \in \mathcal{S}}\left(y_{i}-\sum_{i \in \mathcal{S}} y_{i} / N\right)^{2}\right]}\right\}^{N} .
$$

Consider the square root of the second term defined within the brackets of equation (24) and multiplying 
and dividing the denominator by $\sqrt{2(N-1)}$ yields,

$$
\begin{aligned}
& =\frac{\sqrt{N / 2}\left(\sum_{i \in \mathcal{S}} y_{i} / N-\sum_{i \in \mathcal{S}} x_{i} / N\right)}{\sqrt{2(N-1)} \sqrt{\left[\sum_{i \in \mathcal{S}}\left(x_{i}-\sum_{i \in \mathcal{S}} x_{i} / N\right)^{2}+\sum_{i \in \mathcal{S}}\left(y_{i}-\sum_{i \in \mathcal{S}} y_{i} / N\right)^{2}\right] /\{2(N-1)\}}} \\
& =\frac{\xi}{\sqrt{2(N-1)}} \\
& \approx \frac{\xi}{\sqrt{2 N}}, \text { for } N \gg 1
\end{aligned}
$$

where $\xi$ has the $t$-distribution with $2 N$ degrees of freedom when $\mathcal{H}_{0}$ is true. Hence, equation (23) becomes,

$$
\mathcal{L}(\mathbf{x}, \mathbf{y})=\left\{1+\frac{\xi^{2}}{2 N}\right\}^{N} .
$$

Likelihood ratio, $\mathcal{L}(\mathbf{x}, \mathbf{y})$, as defined in equation (26) is a monotonic function of $\xi^{2}$ and hence a test utilising $\xi^{2}$ must be equivalent to a test utilising $\mathcal{L}(\mathbf{x}, \mathbf{y})$ as the test statistic. From equation (26), it is clear that $\mathcal{L}(\mathbf{x}, \mathbf{y})=1$ when $\xi=0$ and $\xi \longrightarrow \infty$ as $\mathcal{L}(\mathbf{x}, \mathbf{y}) \longrightarrow \infty$. As the mean of the received signal is always positive and since $\xi^{2}$ is a monotonic function, the test statistic of $t$-sensing for $N \gg 1$ can be written as

$$
\theta_{t}=\frac{\sum_{i \in \mathcal{S}} y_{i}-\sum_{i \in \mathcal{S}} x_{i}}{\sqrt{\sum_{i \in \mathcal{S}}\left(x_{i}-\sum_{i \in \mathcal{S}} \frac{x_{i}}{N}\right)^{2}+\sum_{i \in \mathcal{S}}\left(y_{i}-\sum_{i \in \mathcal{S}} \frac{y_{i}}{N}\right)^{2}}} .
$$

Lemma 1. For large numbers of received signal samples $N$, the t-sensing test statistic is asymptotically Gaussian with zero mean and unit variance.

Proof: See Appendix A.

Based on Lemma 1, the threshold $\lambda_{t}$ can be calculated easily by specifying target probability of false alarm $\widetilde{P}_{f}$ using

$$
\lambda_{t}=\Phi^{-1}\left(1-\widetilde{P}_{f}\right)
$$

1) Asymptotic Non-parametric behavior of t-sensing: The test statistic of $t$-sensing is derived in equation (24) under the assumption of Gaussian noise, so the probability of false alarm, in general, will not remain constant for non-Gaussian noise. However $\theta_{t}$ shows interesting asymptomatic properties. For large values of $N$, the quantities $\sum x_{i} / N$ and $\sum y_{i} / N$ are asymptotically Gaussian by applying the 
central limit theorem, hence their difference is also Gaussian distributed with zero mean and variance given by $\widehat{\varphi}^{2}$, where

$$
\widehat{\varphi}^{2}=\sqrt{\frac{1}{2 N} \sum_{i \in \mathcal{S}}\left[y_{i}-\mu_{\mathbf{y}}\right]^{2}+\frac{1}{2 N} \sum_{i \in \mathcal{S}}\left[x_{i}-\mu_{\mathbf{x}}\right]^{2}},
$$

and $\mu_{\mathbf{x}}$ and $\mu_{\mathbf{y}}$ are defined as,

$$
\begin{aligned}
\mu_{\mathbf{y}} & =\frac{1}{N} \sum_{i \in \mathcal{S}} y_{i} \\
\mu_{\mathbf{x}} & =\frac{1}{N} \sum_{i \in \mathcal{S}} x_{i}
\end{aligned}
$$

Since the $t$-sensing test statistic is asymptotically Gaussian, if the threshold is chosen according to (28) the probability of false alarm will remain constant for any noise distribution. Hence, the proposed $t$-sensing is asymptotically non-parametric.

2) Probability of miss detection for t-sensing: Probability of miss detection for $t$-sensing is

$$
\begin{aligned}
P_{m} & =1-\operatorname{Prob}\left\{\theta_{t}>\lambda_{t} \mid \mathcal{H}_{1}\right\}, \\
& =F\left(\theta_{t} \mid \mathcal{H}_{1} ; \lambda_{t}\right),
\end{aligned}
$$

where $F\left(\theta_{t} \mid \mathcal{H}_{1} ; \lambda_{t}\right)$ is the CDF of test statistic $\theta_{t}$. In order to calculate the probability of miss detection, one needs to calculate the distribution function of the $t$-sensing test statistic. The actual distribution of $\theta_{t} \mid \mathcal{H}_{1}$ is dependent on the unknown variances under the two hypothesis $\mathcal{H}_{0}$ and $\mathcal{H}_{1}$ and cannot be expressed analytically (Behrens-Fisher problem) [14].

Lemma 2. The distribution of the t-sensing test statistic, given $\mathcal{H}_{1}$, can be approximated by a chi-square distribution with degrees of freedom given by

$$
\Lambda_{\theta_{t} \mid \mathcal{H}_{1}}=(N-1)\left[1+\frac{2 \varphi_{\mathbf{y}}^{2} \varphi_{\mathbf{x}}^{2}}{\varphi_{\mathbf{y}}^{4}+\varphi_{\mathbf{x}}^{4}}\right]
$$

where

$$
\begin{aligned}
\varphi_{\mathbf{y}}^{2} & =\frac{1}{N} \sum_{i \in \mathcal{S}}\left[y_{i}-\mu_{\mathbf{y}}\right]^{2} \\
\varphi_{\mathbf{x}}^{2} & =\frac{1}{N} \sum_{i \in \mathcal{S}}\left[x_{i}-\mu_{\mathbf{x}}\right]^{2}
\end{aligned}
$$

Proof: According to equation (27), $t$-sensing test statistic is the linear combination of independent sample variances. We start the proof by considering a random sample $u=\left[u_{1}, u_{2}, \cdots, u_{N}\right]^{T}$ of size $N$ from a Gaussian distribution defined as $\mathcal{N}\left(\bar{\mu}, \overline{\sigma^{2}}\right)$, then define

$$
\varrho^{2}=\left[\frac{1}{N-1}\right] \sum_{i \in \mathcal{S}}\left(u_{i}-\bar{u}\right)^{2}=\left[\frac{\overline{\sigma^{2}}}{N-1}\right] \sum_{i \in \mathcal{S}}\left(\frac{u_{i}-\bar{u}}{\bar{\sigma}}\right)^{2},
$$


where $\bar{u}=\frac{1}{N} \sum_{i \in \mathcal{S}} u_{i}$. It is well known that the sum of the square of Gaussian random numbers follows a chi-square distribution so equation (36) can be written as

$$
\varrho^{2} \sim\left[\frac{\overline{\sigma^{2}}}{N-1}\right] \chi_{(N-1)}^{2},
$$

where $\chi_{n}^{2}$ is the chi-square distribution with $n$ degrees of freedom.

$$
\begin{aligned}
\mathrm{E}\left[\varrho^{2}\right] & =\left[\frac{\overline{\sigma^{2}}}{N-1}\right](N-1)=\overline{\sigma^{2}} \\
\operatorname{Var}\left(\varrho^{2}\right) & =\left[\overline{\overline{\sigma^{2}}}\right]^{2} 2(N-1)=\frac{2 \overline{\sigma^{4}}}{N-1}
\end{aligned}
$$

where $\mathrm{E}[$.$] and \operatorname{Var}($.$) represent mean and variance operation, respectively. Define,$

$$
\Omega^{2}=\alpha_{1} \times \varrho_{1}^{2}+\alpha_{2} \times \varrho_{2}^{2}=\sum_{\kappa \in\{\mathbf{x}, \mathbf{y}\}} \alpha_{\kappa} \varrho_{\kappa}^{2}
$$

where $\alpha_{\kappa}$ are known constants. Now we can calculate the variance of $\Omega^{2}$ as

$$
\operatorname{Var}\left(\Omega^{2}\right)=\sum_{\kappa \in\{\mathbf{x}, \mathbf{y}\}} \alpha_{\kappa}^{2} \frac{2 \varrho_{\kappa}^{2}}{N-1}
$$

If we want to approximate the distribution of $\Omega^{2}$ by chi-square distribution with degrees of freedom $\Lambda$, $\chi_{\Lambda}^{2}$ will have the same expected value and variance. Hence, from equation (38)

$$
\mathrm{E}\left[\chi_{\Lambda}^{2}\right]=\mathrm{E}\left[\Omega^{2}\right]=\sum_{\kappa \in\{\mathbf{x}, \mathbf{y}\}} \alpha_{\kappa} \overline{\sigma_{\kappa}^{2}}
$$

The variance of the standard chi-square random variable is given by

Equating (39) and (43),

$$
\begin{aligned}
\operatorname{Var}\left(\chi_{\Lambda}^{2}\right) & =\frac{2 \times \mathrm{E}\left[\chi_{\Lambda}^{2}\right]^{2}}{\text { degrees of freedom }} \\
& =\frac{2}{\Lambda}\left(\sum_{\kappa \in\{\mathbf{x}, \mathbf{y}\}} \alpha_{\kappa} \overline{\sigma_{\kappa}^{2}}\right)
\end{aligned}
$$

$$
\Lambda=\frac{\left(\sum_{\kappa \in\{\mathbf{x}, \mathbf{y}\}} \alpha_{\kappa} \overline{\sigma_{\kappa}^{2}}\right)^{2}}{\sum_{\kappa \in\{\mathbf{x}, \mathbf{y}\}} \alpha_{\kappa}^{2} \frac{\varrho_{\kappa}^{4}}{N-1}} .
$$

Substituting $\sigma=\varphi$ and $\alpha=1 / N$ in equation (44) yields

$$
\begin{aligned}
\Lambda_{\theta_{t} \mid \mathcal{H}_{1}}=\Lambda & =\frac{(N-1)\left(\varphi_{\mathbf{y}}^{2}+\varphi_{\mathbf{x}}^{2}\right)^{2}}{\varphi_{\mathbf{y}}^{4}+\varphi_{\mathbf{x}}^{4}} \\
& =(N-1)\left[1+\frac{2 \varphi_{\mathbf{y}}^{2} \varphi_{\mathbf{x}}^{2}}{\varphi_{\mathbf{y}}^{4}+\varphi_{\mathbf{x}}^{4}}\right] .
\end{aligned}
$$


Hence proved.

Based on lemma 2, the distribution function of $\theta_{t}$ given $\mathcal{H}_{1}$ can be derived and the probability of miss detection defined in equation (32) is written as

$$
\begin{aligned}
P_{m} & =F\left(\theta_{t} \mid \mathcal{H}_{1} ; \lambda_{t}\right) \\
& =\frac{\Gamma\left(\Lambda / 2, \lambda_{t} / 2\right)}{\Gamma(\Lambda / 2)}
\end{aligned}
$$

To summarise, the procedure for applying the $t$-sensing algorithm to the problem of spectrum sensing is as follows: before the start of a sensing session, collect noise samples $\mathbf{x}$ (depending on noise dynamics the noise observation vector can be updated after a few sensing sessions) and store them in the internal memory. Receive the observation vector $\mathbf{y}$ on the sensing channel and calculate the test statistic $\theta_{t}$ using equation (27). Calculate the decision threshold using equation (28) and compare with $\theta_{t}$ to decide if the transmit signal is present or absent.

\section{Simulation Results}

In this section, simulation results are presented evaluating the performance of the proposed sensing algorithms described in section III. We compare AD sensing, KS sensing and $t$-sensing and use ED sensing as a reference. We performed extensive Monte-Carlo simulations and compare the results using ROC curves. We assumed $\sigma_{n}=1, C=1, N=32$ and $\mathrm{SNR}=-2 \mathrm{~dB}$ in all simulation results unless otherwise stated.

In [6], it was shown by simulations that $\mathrm{AD}$ sensing performs much better than $\mathrm{ED}$ sensing at low SNR. The signal model used in [6] is quite simple and under both hypothesis $\mathcal{H}_{0}$ and $\mathcal{H}_{1}$ the received signal variance remains the same. It is interesting to note that with the system model defined in [6], the spectrum sensing problem can be formulated as a location testing problem and hence the less complex $t$-sensing performs better than $\mathrm{AD}$ sensing. This is confirmed by our simulations as shown in Fig. 1, where the same parameters and model described in [6] were used. The AD sensing method is difficult to implement in real time even with a fast processor; while on the other hand $t$-sensing test statistics and threshold calculations are straight-forward.

Fig. 2 depicts the complementary ROC curves in the low SNR region; that is, the received SNR is $-2 \mathrm{~dB}$ and the number of received samples are 32 . Here, we assume that the channel is Rician fading and the noise process is modeled as a Gaussian process with zero mean and variance $\sigma_{n}^{2}$. It is clear that $t$-sensing outperforms ED, AD and KS based spectrum sensing at low SNR. For example, at $P_{f}=10^{-2}$, the miss detection probability for $t$-sensing is $0.48 \%$, while for the case of energy detection the probability 


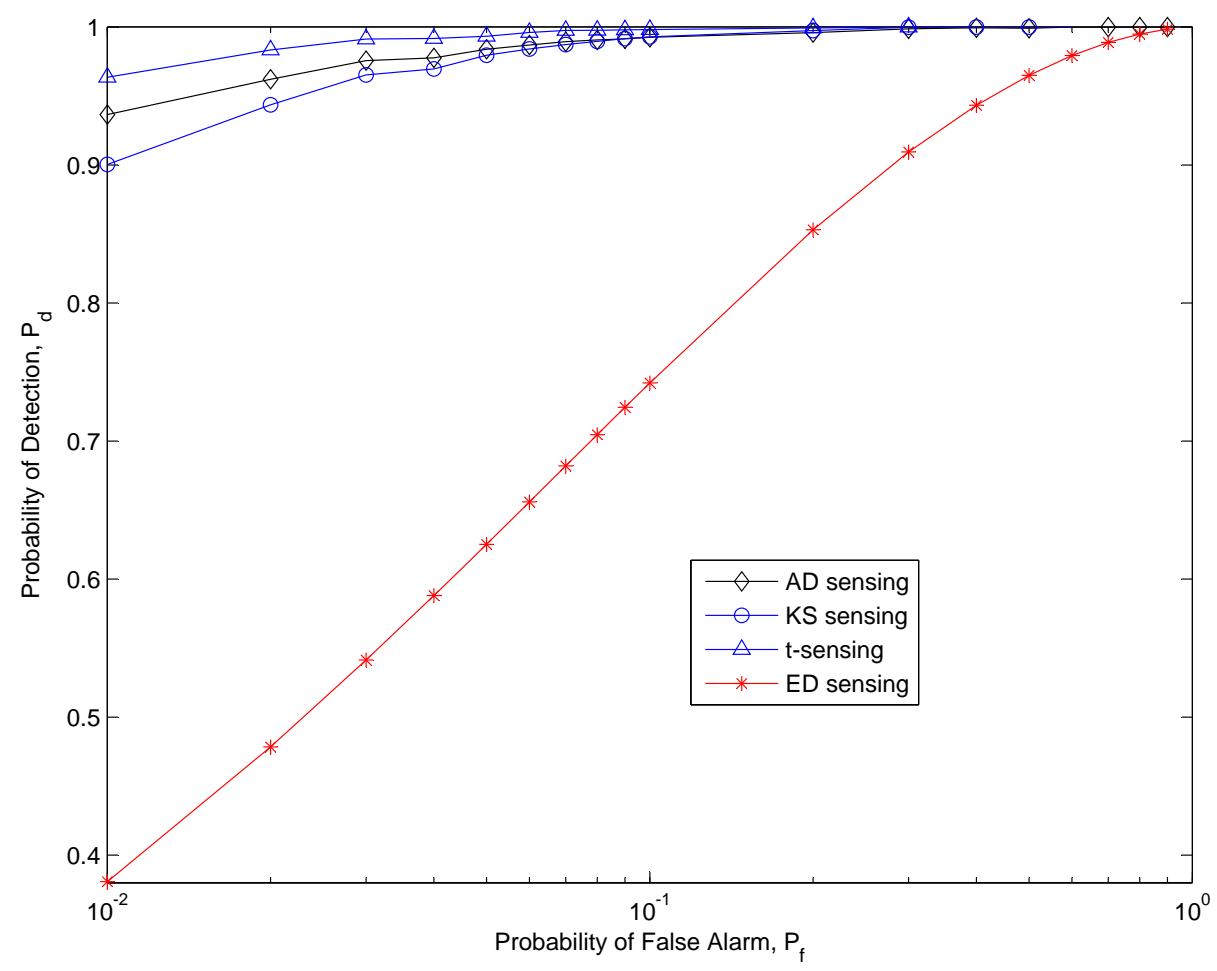

Fig. 1. ROC curves with system model used in [6], $N=28$ and SNR $=-2 \mathrm{~dB}$

of miss detection is $47.69 \%$. The miss detection probability of AD sensing and KS sensing at $P_{f}=10^{-2}$ is around $6 \%$ and $41 \%$ respectively. Despite the fact that $\mathrm{AD}$ sensing and $\mathrm{KS}$ sensing require a-priori information about the noise distribution, their performance is inferior compared to $t$-sensing in Gaussian noise.

Fig. 3 shows the miss detection probability versus received SNR for a given false alarm probability, $\widetilde{P}_{f}=10^{-1}$, in a Rician fading channel. As seen from Fig. 3 in order to detect signals as low as $-15 \mathrm{~dB}$, miss detection probability of $t$-sensing, AD sensing, KS sensing and energy detection is $32.63 \%, 76.38 \%$, $79.55 \%$ and $86.58 \%$, respectively. Fig. 4 shows the detection performance of the sensing schemes versus the $\mathrm{K}$ factor of the Rician channel. With increasing $\mathrm{K}$ factor the detection performance increases as the distance between two distributions under $\mathcal{H}_{0}$ and $\mathcal{H}_{1}$ increases.

As discussed in section III-C, the $t$-sensing scheme is also robust asymptotically and gives similar performance if the noise is non-Gaussian. To show the robustness of our proposed method, we consider another type of noise commonly present in communication systems, it should be noted that the conclusions remain valid for any other type of noise as well. Recent studies have shown that non-Gaussian noise is 


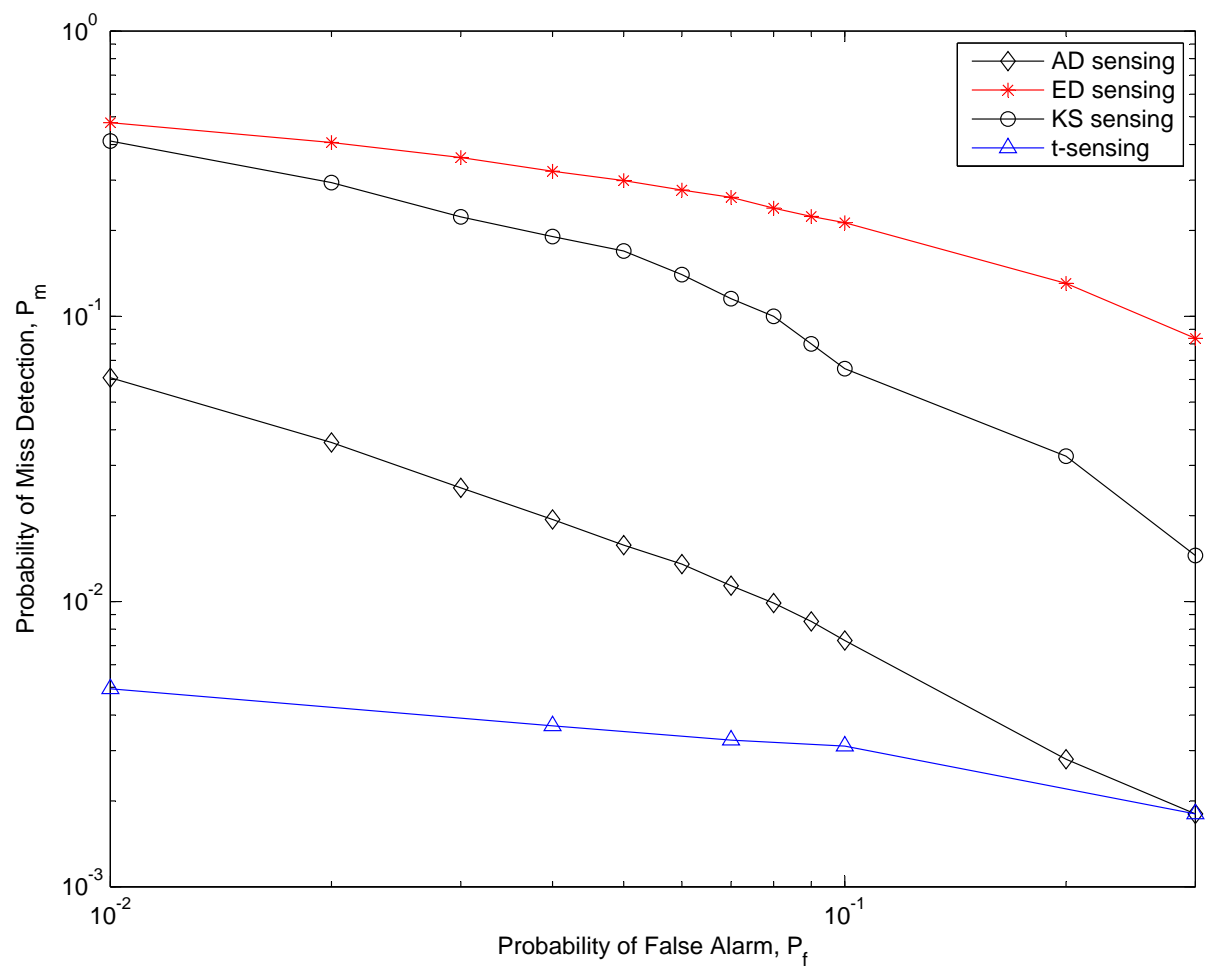

Fig. 2. ROC curves in Rician Fading channel with Samples $=32, C=1, K=10$ and $S N R=-2 d B$

present in indoor environments and Laplace distribution is widely used for modelling such noise [15]. To see how $t$-sensing performs under Laplacian noise, we plot the ROC curves with with sample size $N=32$, and SNR $-10 \mathrm{~dB}$, in Fig. 6. Although $\theta_{t}$ is derived for Gaussian noise, it gives similar performance in non-Gaussian noise as can be seen in Fig. 6. An interesting observation in Fig. 6 is that the asymptomatic behavior can be seen even for $N=32$, our simulation study shows that $t$-sensing is robust for $N \geq 25$. However, as indicated in section I, the exact noise distribution is often unknown in real situations and $\mathrm{AD}$ and $\mathrm{KS}$ based sensing work only if the exact noise distribution is known a-priori. Hence, under such conditions $t$-sensing is the most suitable choice.

\section{Conclusions}

In this paper, spectrum sensing algorithms based on statistical tests are discussed and KS sensing and $t$-sensing are proposed. The efficacy of the proposed sensing schemes has been demonstrated through simulations. The simulation results show that the proposed $t$-sensing outperforms energy-detection-based spectrum and AD sensing as proposed in literature, and they introduce less complexity. It is thus concluded 


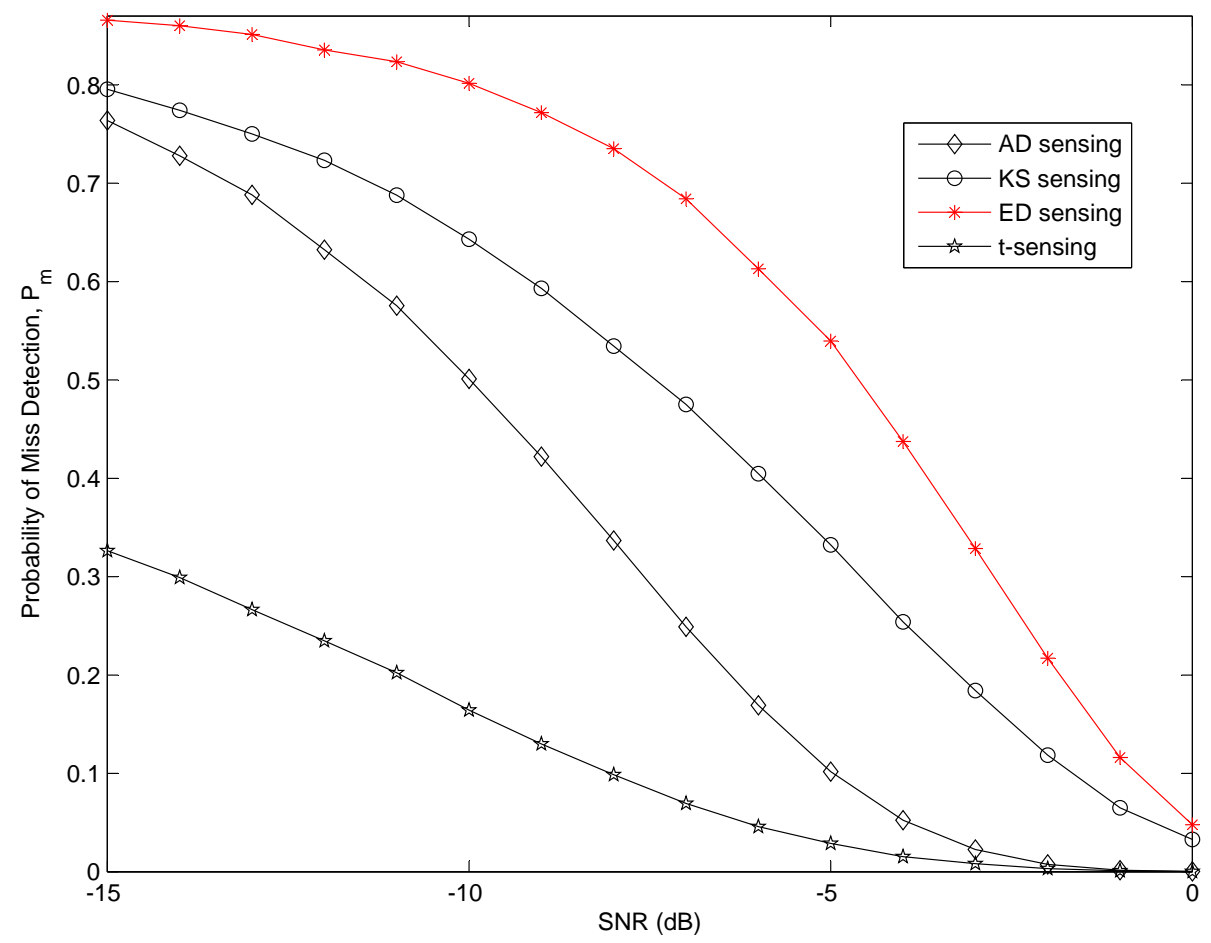

Fig. 3. $P_{m}$ versus SNR in Rician Fading channel with Samples $=32, \mathrm{C}=1, \mathrm{~K}=10$ and $P_{f}=10^{-1}$

in this paper that, if the noise distribution is known and it is Gaussian then $t$-sensing is superior in terms of detection probability, while with known non-Gaussian noise distribution KS sensing is the better alternative. We further emphasise the fact that the proposed $t$-sensing is robust asymptotically and gives similar performance for an unknown non-Gaussian noise distribution while AD and KS sensing are applicable only if the exact noise distribution is known. As future work, it would be of interest to quantify the complexity of all algorithms and to implement all sensing techniques on hardware platforms.

\section{ACKNOWLEDGMENT}

The research leading to these results was derived partially from the European Community's Seventh Framework Programme (FP7) under Grant Agreement number 248454 (QoSMOS) and partially from the University of Greenwich Research \& Enterprise Investment Programme grant under agreement number RAE-ENG-04/12. 


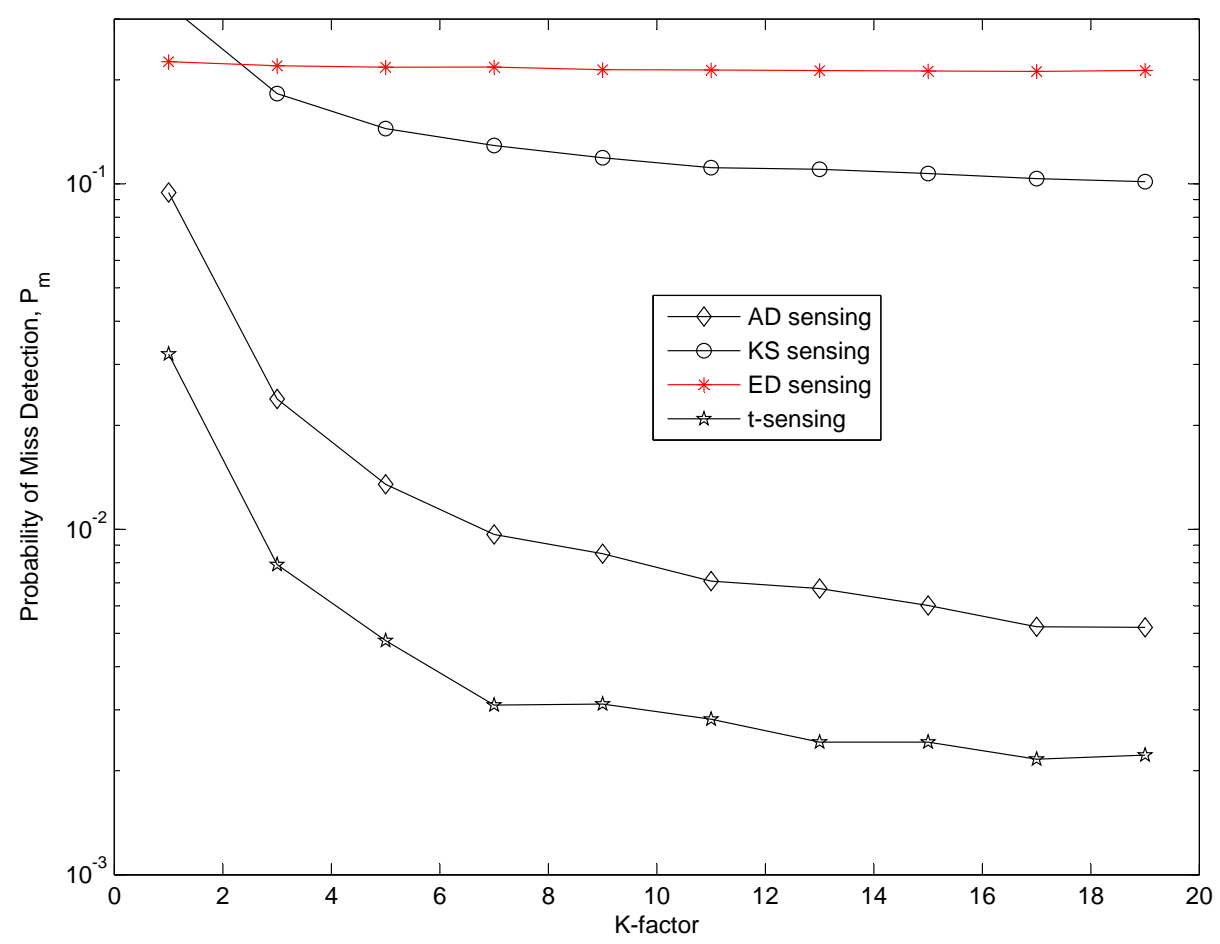

Fig. 4. $P_{m}$ versus $\mathrm{K}$ factor with Samples $=32, \mathrm{C}=1, \mathrm{~K}=10$ and $P_{f}=-10^{-1}$

\section{APPENDIX A}

\section{Asymptotic Distributions of $t$-SENSING Test STATISTIC}

Define the mean and variance of received signal samples in a sensing channel of bandwidth $W$ as

$$
\begin{aligned}
& \mu_{\mathbf{y}}=\frac{1}{N} \sum_{i \in \mathcal{S}} y_{i} \\
& \varphi_{\mathbf{y}}^{2}=\frac{1}{N} \sum_{i \in \mathcal{S}}\left[y_{i}-\mu_{\mathbf{y}}\right]^{2}
\end{aligned}
$$

Similarly, denote the mean and variance of noise samples $\mathrm{x}$ as

$$
\begin{aligned}
& \mu_{\mathbf{x}}=\frac{1}{N} \sum_{i \in \mathcal{S}} x_{i} \\
& \varphi_{\mathbf{x}}^{2}=\frac{1}{N} \sum_{i \in \mathcal{S}}\left[x_{i}-\mu_{\mathbf{x}}\right]^{2}
\end{aligned}
$$

Substituting equations (A-1) and (A-2) in equation (27)

$$
\theta_{t}=\frac{\sqrt{\frac{N}{2}}\left[\mu_{\mathbf{y}}-\mu_{\mathbf{x}}\right]}{\sqrt{\left[\varphi_{\mathbf{x}}^{2}+\varphi_{\mathbf{y}}^{2}\right] / 2}}
$$




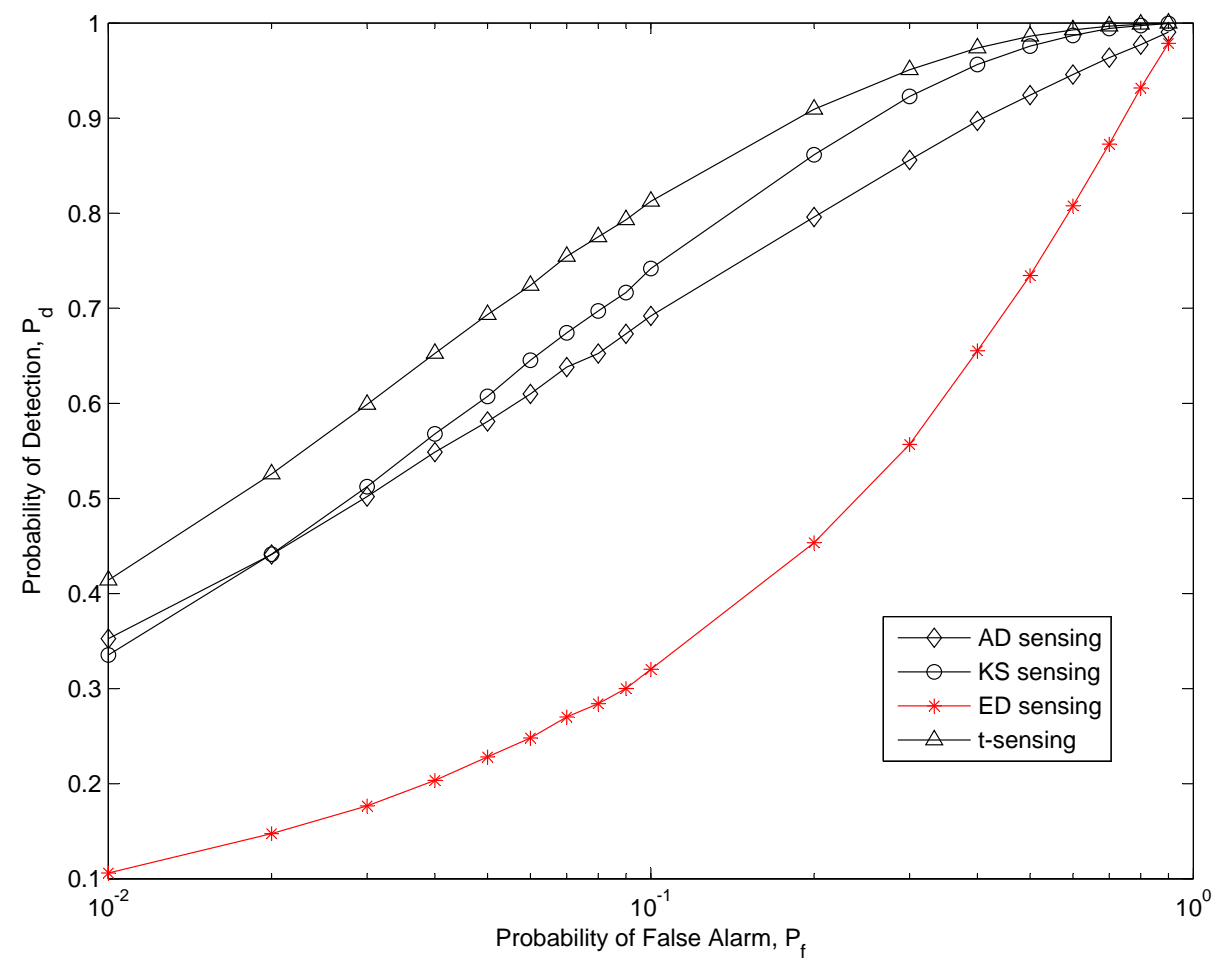

Fig. 5. ROC curves [Gaussian Noise] with Samples $=32$ and $\mathrm{SNR}=-10 \mathrm{~dB}$

The quantity in the denominator of equation (A-3) is the estimate of the variance and is denoted by $\widehat{\varphi}^{2}$, hence

$$
\theta_{t}=\frac{\mu_{\mathbf{y}}-\mu_{\mathbf{x}}}{\sqrt{\frac{2}{N} \widehat{\varphi}^{2}}} .
$$

The quantity $\mu_{\mathbf{y}}-\mu_{\mathbf{x}}$ in equation (A-4) is normal distributed since both $\mathbf{x}$ and $\mathbf{y}$ are normal distributed. Under $\mathcal{H}_{0}, \mu_{\mathbf{y}}-\mu_{\mathbf{x}}$ tends to zero while under $\mathcal{H}_{1}$ the numerator of equation (A-4) approaches $C \mu_{h}$; Similarly, for large values of $N$ the quantity $\mu_{\mathbf{y}}-\mu_{\mathbf{x}}$ has a variance equal to $\frac{2}{N} \sigma_{n}^{2}$. Since, for large values of $N$, the denominator of equation (A-4) approaches $\frac{2}{N} \sigma_{n}, \theta_{t}$ is asymptotically Gaussian with certain mean and variance 1 by the central limit theorem.

\section{REFERENCES}

[1] K. Arshad, M. A. Imran, and K. Moessner, "Collaborative Spectrum Sensing Optimisation Algorithms for Cognitive Radio Networks," International Journal of Digital Multimedia Broadcasting, vol. 2010, no. 424036, 2010.

[2] Federal Communications Commission: Second Memorandum Opinion and Order, 2010. [Online]. Available: http://www.fcc.gov/ 


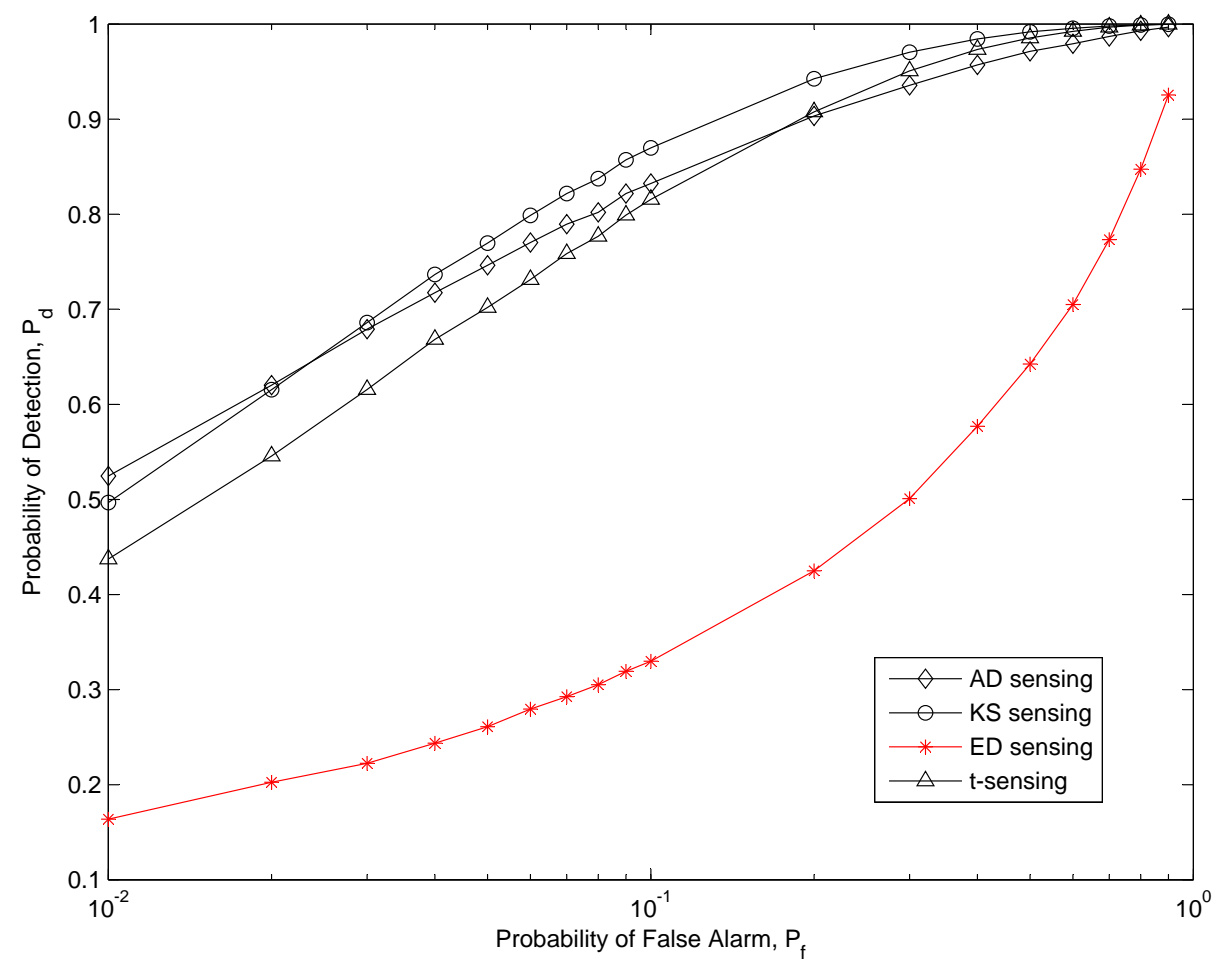

Fig. 6. ROC curves [Laplacian Noise] with Samples $=32$ and SNR $=-10 \mathrm{~dB}$

[3] FCC Auctions March 2008, accessed July 2009. [Online]. Available: http://www.wireless.fcc.gov/

[4] V. Goncalves and S. Pollin, "The value of sensing for TV White Spaces," in IEEE Symposium on New Frontiers in Dynamic Spectrum Access Networks (DySPAN), may 2011, pp. $231-241$.

[5] T. Yucek and H. Arslan, "A Survey of Spectrum Sensing Algorithms for Cognitive Radio Applications," IEEE Communications Surveys \& Tutorials, vol. 11, no. 1, pp. 116-130, 2009.

[6] H. Wang, E.-H. Yang, Z. Zhao, and W. Zhang, "Spectrum Sensing in Cognitive Radio using Goodness of Fit testing," IEEE Transactions on Wireless Communications, vol. 8, no. 11, pp. 5427-5430, November 2009.

[7] J. Proakis, Digital Communications. McGrawHill, 2000, vol. 4th.

[8] G. Zhang, X. Wang, Y.-C. Liang, and J. Liu, "Fast and robust spectrum sensing via kolmogorov-smirnov test," Trans. Comm., vol. 58, pp. 3410-3416, December 2010. [Online]. Available: http://dx.doi.org/10.1109/TCOMM.2010.11.090209

[9] H. Urkowitz, "Energy Detection of Unknown Deterministic Signals," IEEE Proceedings, vol. 55, no. 4, pp. 523-531, April 1967.

[10] T. W. Anderson and D. A. Darling, "Asymptotic Theory of Certain "Goodness of Fit" Criteria Based on Stochastic Processes," Annals of Mathematical Statistics, vol. 23, no. 2, pp. 193-212, 1952.

[11] M. A. Stephens, "EDF Statistics for Goodness of Fit and Some Comparisons," Journal of the American Statistical Association, vol. 69, no. 347, pp. 730-737, 1974.

[12] F. Massey, "The Kolmogorov-Smirnov Test for Goodness of Fit," Journal of the American Statistical Association, vol. 46, 
no. 253 , pp. $68-79,1951$.

[13] J. Carlyle and J. Thomas, "On Nonparametric Signal Detectors," IEEE Transactions on Information Theory, vol. 10, no. 2, pp. 146-152, 1963.

[14] S.-H. Kim and A. S. Cohen, "On the Behrens-Fisher Problem: A Review," Journal of Educational and Behavioral Statistics, vol. 23 , no. 4, pp. 356-377, Winter 1998.

[15] R. Marks, G. Wise, D. Haldeman, and J. Whited, "Detection in Laplace Noise," IEEE Transactions on Aerospace and Electronic Systems, vol. AES-14, no. 6, pp. 866 -872, nov. 1978. 